\title{
Search for X-ray quasi-periodic oscillations with weighted wavelet z-transform technique
}

\author{
Xiaopan Li \\ College of Physics and Electronic Engineering, Zhaotong University, Zhaotong 657000, China \\ Ixpzrc@163.com
}

Keywords: WWZ, periodicity, wavelet, PKS 0537-441.

\begin{abstract}
The searching for the quasi-periodic oscillations (QPOs) of some Active galactic nuclei (AGNs) plays an important role in understanding the physical mechanism and theoretical model of AGNs. The AGN PKS 0537-441 was monitored in the X-ray $15-50 \mathrm{keV}$ bands in 2005-2016 by the Swift/BAT instruments, which provide an opportunity to identify the QPOs in X-ray band. By mean of the weighted wavelet z-transform technique (WWZ), QPOs of 66, 198 and 398 day are identified. Moreover, the instabilities in the accretion flow might be responsible the QPOs in the X-ray band.
\end{abstract}

\section{Introduction}

Active galactic nuclei (AGNs) have been a research hotspot for several years, which presumably possess black holes with masses of $10^{6} \sim 10^{10} \mathrm{M}_{\odot}$ and show significant light variability. Characteristic timescales of variability, from minutes through days and months to decades, provide an important way to study the physical mechanism and theoretical model of the its central regions [1]. Different variability timescales indicating different emission mechanism have been widely studied by many researchers and occasionally demonstrate periodic variations in some AGNs. The searching for the quasi-periodic oscillations (QPOs) of some AGNs at different frequencies reveals that the characteristic timescales of QPOs are generally diverse and can be divided into three classed, e.g., short-term class with timescales from minutes to days, medium-term class from weeks to months, and long-term class cover years to decades[2].

The AGN PKS 0537-441 belongs to the blazar which is a subclass of AGNs, is one of the most famous southern hemisphere blazar. This luminous source, at red-shift $z=0.896$, has been extensively monitored across the overall electromagnetic spectrum and shows significant variability on diverse timescales, ranging form minutes to months. In the near-infrared and optical band, a short-term timescale of about 25 minutes has been identified by Impiombato et al. and Zhang et al.[3,4]. In the $\mathbf{J}$ and V band, respectively. Recently, Sandrinelli et al. detected a QPO of about 140 days in the optical $\mathrm{R}$ band with the data obtained from ESO/REM and Yale/SMARTS telescopes, and a QPO of 280 days in the gamma-ray with the Fermi/LAT instrument [5]. However, the timescales of QPOs of PKS 0537-441 in other wavebands are still misty and waiting for probing.

In this paper, the X-ray QPOs of PKS 0537-441 have been discussed. Section 2 gives the description of the observation, Section 3 the method, Section 4 the searching for QPOs and Section 5 the conclusion.

\section{Observations}

The observations data we analyzed are taken form the public database of the Swift Gamma-Ray Burst (GRB) explorer project. The Swift satellite rocketed in 2004 carries three monitors, i.e., the Burst Alert Telescope (BAT, 15-150 keV), the X-ray Telescope (XRT, 0.2-10.0 keV) and the Ultraviolet/Optical Telescope (UVOT, 170-600 nm), which have created an infusion of scientific discovery, particularly in high energy environments. In spite of the main research target is study GRB, the Swift satellite has been performed to other transient or variable phenomena and became extremely useful for studies of blazars. 


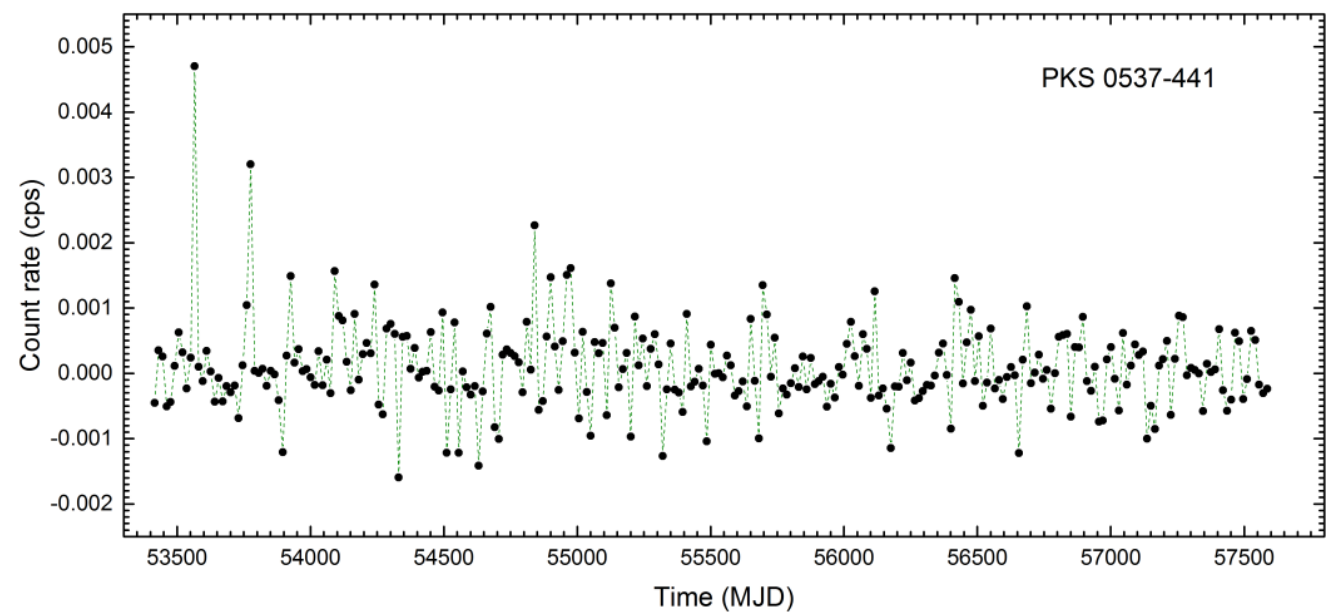

Fig. 1 Long-term light curve of PKS 0537-441 in the X-ray 15-50 keV band.

As a hard X-ray telescope on board Swift, BAT has a large filed of view and covers the energy range from 15 to $150 \mathrm{keV}$. Since 2005, PKS 0537-441 was monitored by the BAT instrument at 15-150 keV energies. Moreover, the BAT/team performs the basic analysis to the SCALED MAPS of the BAT monitor and publishes the BAT Transient light curves of hundreds sources in a single energy band (15-50 keV) publicly, which provides us an opportunity to study the QPOs of PKS 0537-441. More details about the BAT and BAT/team refer to Krimm et al [6]. The long-term light curve in $15-50 \mathrm{keV}$ band is shown in Fig. 1. The light curve shows significant variations and periodic fluctuations phenomenon. Therefore, technical analysis method like wavelet technique is used to search the QPOs of this source.

\section{Weighted wavelet z-transform technique}

The wavelet technique is useful for providing time and frequency resolution simultaneously and measuring the local timescale of the data series. As a modified wavelet technique, weighted wavelet $z$-transform technique (WWZ)[7] take the abbreviated Morlet wavelet

$$
f(z)=e^{i w(t-\tau)-c w^{2}(t-\tau)^{2}}
$$

to the data series $\left\{x\left(t_{\alpha}\right), \alpha=1,2, \ldots, n\right\}$, and project the data onto the complex trial function of $e^{i \omega(t-\tau)}$ with the real statistical weights $\omega_{\alpha}=e^{-c \omega^{2}\left(t_{\alpha}-\tau\right)^{2}}$, where $\omega$ is the frequency, $\tau$ the time shift and $c$ the constant. The the WWZ is defined as

$$
\mathrm{WWZ}=\frac{(N-3) V_{y}}{2\left(V_{x}-V_{y}\right)}
$$

Where $N$ is the effective number, $V_{x}$ and $V_{y}$ are the weighted variations of the uneven data $x$ and the model function $y$, respectively. These factors can be expressed as

$$
\begin{aligned}
& N=\frac{\left(\sum \omega_{\alpha}\right)^{2}}{\sum \omega_{\alpha}^{2}}=\frac{\left[\sum e^{-c \omega^{2}\left(t_{\alpha}-\tau\right)^{2}}\right]^{2}}{\sum e^{-2 c \omega^{2}\left(t_{\alpha}-\tau\right)^{2}},} \\
& V_{x}=\frac{\sum_{\alpha} \omega_{\alpha} x^{2}\left(t_{\alpha}\right)}{\sum_{\lambda} \omega_{\lambda}}-\left[\frac{\sum_{\alpha} \omega_{\alpha} x\left(t_{\alpha}\right)}{\sum_{\lambda} \omega_{\lambda}}\right]^{2}=\langle x \mid x\rangle-\langle 1 \mid x\rangle^{2}, \\
& V_{x}=\frac{\sum_{\alpha} \omega_{\alpha} y^{2}\left(t_{\alpha}\right)}{\sum_{\lambda} \omega_{\lambda}}-\left[\frac{\sum_{\alpha} \omega_{\alpha} y\left(t_{\alpha}\right)}{\sum_{\lambda} \omega_{\lambda}}\right]^{2}=\langle y \mid y\rangle-\langle 1 \mid y\rangle^{2} .
\end{aligned}
$$

WWZ has been wildly used to detected the time and frequency and measure the local timescale of the data series. More details about the parameters of WWZ refer to Foster [7]. 


\section{QPOs of PKS 0537-441}

The WWZ analysis for the entire X-ray band observations of PKS 0537-441 is shown in the Fig. 2. The minimum period is set as 15 days because our data are 15 days binned, while maximum period is set as 100 days corresponds to nearly five complete cycles during the whole time interval of $4184 \mathrm{~d}$. A period step of one day and a decay constant $\mathrm{c}=0.00125$ were adopted to identify the possible periodicities.

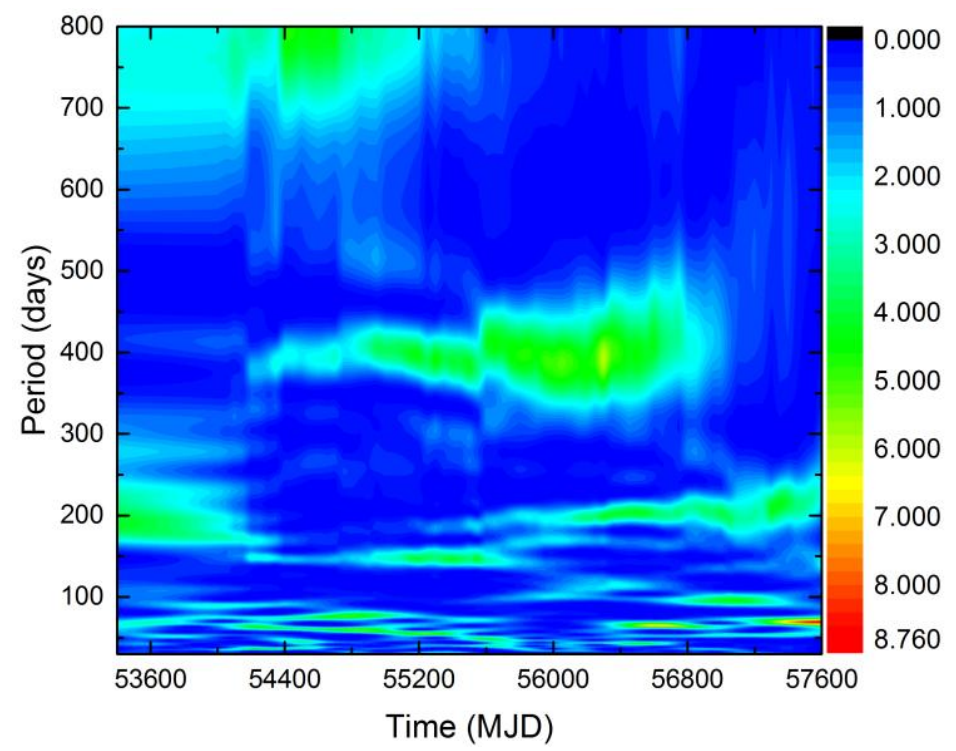

Fig. 2 The WWZ statistic as a function of the time and period, the color of the contours in WWZ map represent the significance of WWZ statistic

In the WWZ map (Fig. 2), there are at least three strong signals which represent periodicities of about 70, 200 and 400 day in the contour, indicating a complicated optical variability behavior of PKS 0537-441. The WWZ analysis reveals the primary periodicity of about 400 day appeared during the period from MJD (Modified Julian Date) 54400 to MJD 56800 approximately. Another significant signal appears at around 200 day, which indicate a possible periodicity of about 200 day. This periodic signal seems to be existed in the whole observation run, although it shows a fluctuating feature around 200 day in amplitude and period during the period from MJD 54000 to MJD 55800. From the WWZ map, we can find that in the period range of 50-70 day, a remarkable periodicity exists although the signal seems to fluctuated obviously. The presences of possible QPOs is not continuous along the time axis and show diverse fluctuation in periods, which indicate that the periodical feature is complex and likely unstable.

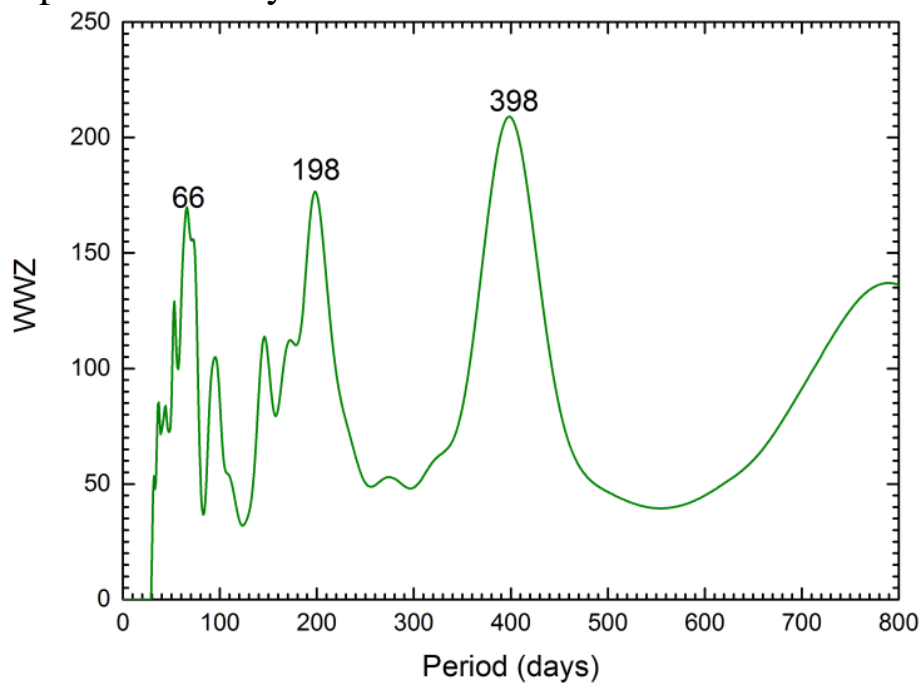

Fig. 3 The integrated WWZ power spectrum.

In order to determine the major period of WWZ map, the integrated WWZ power spectrum was calculated and presented in Fig. 3. The WWZ power spectrum demonstrate statistically the 
significance and amplitudes of the multiply periodicities at each characteristic period. As can be seen from the integrated power spectrum, three significant peaks marked with numbers is preferentially occurs at 66, 198 and 398 day, which reveals that the QPOs of 66, 198 and 398 day exhibited in the $\mathrm{X}$-ray light curve.

In order to interpret the QPOs in AGNs, researchers proposed many models, such as the binary super massive black hole system (BBH) and the global p-mode oscillations in a thick accretion disk. However, considering that the QPOs of 66, 198 and 398 day are longer than a week and shorter than several years, we suggest that the instabilities in the accretion flow should responsible for the QPOs detected in the X-ray light curve while the BBH and thick accretion disk oscillation models are not associated with the QPOs.

\section{Conclusion}

The AGN PKS 0537-441 was monitored in the X-ray 15-50 keV band during the period from 2005 December to 2016 July by the BAT instruments hosted in Swift satellite. The X-ray light curve shows significant variations and periodic fluctuations phenomenon, which provides an important opportunity to search the QPOs of PKS 0537-441. By means of the WWZ technique, periodicities of about 70 day, 200 day and 400 day in the contour map have been confirmed, and the integrated WWZ

power spectrum gives QPOs with timescales of 66, 198 and 398 day exhibited in the X-ray light curve. Finally, I suggest the instabilities in the accretion flow is associated with the QPOs detected in the $\mathrm{X}$-ray light curve.

\section{Acknowledgments}

This work is supported by the Youth Program of the Applied Basic Research Projects of Yunnan Province, China (2013FD056). We acknowledge the use of public data from the Swift data archive.

\section{References}

[1]. D'Ammando F., Antolini E., Tosti G., et al. Long-term monitoring of PKS 0537-441 with Fermi-LAT. Monthly Notices of the Royal Astronomical Society. Vol. 431 (2013) 2481-2492.

[2]. Rani, B., Gupta, A.C., Strigachev, A., et al.. Short-term flux and colour variations in low-energy peaked blazars. Monthly Notices of the Royal Astronomical Society. Vol. 404 (2010) 1992-2007.

[3]. Impiombato, D. Covino, S., Treves, A., et al. Optical and infrared photometry of the blazar PKS 0537-441: longand short timescale variability. Astrophysical Journal Supplement Series. Vol. 192 (2011) 12-17.

[4]. Zhang, B.-K., Wang, S., Zhao, X.-Y., et al. Long-term optical and infrared variability of the BL Lac object PKS 0537-441. Monthly Notices of the Royal Astronomical Society. Vol. 428 (2013) 3630-3635.

[5]. Sandrinelli, A., Covino, S., \& Treves, A. Gamma-ray and optical oscillations in PKS 0537-441. Astrophysical Journal. Vol. 820 (2016) 20-26.

[6]. Krimm, H. A., Holland, S. T., Corbet, R. H. D., et al. the Swift/BAT hard X-ray transient monitor. Astrophysical Journal Supplement Series. Vol. 209 (2013) 14-47.

[7]. Foster, G. Wavelets for period analysis of unevenly sampled time series. Astrophysical Journal. Vol. 112 (1996) 1709-1729. 